\title{
Inteligencia emocional en estudiantes de la Universidad Nacional del Centro del Peru, 2013
}

\author{
Emotional intelligence in students from the Universidad Nacional \\ del Centro del Perú, 2013
}

Cesar Augusto Reyes Lujan', Iris Yone Carrasco Díaz

Universidad Nacional del Centro del Perú

\section{RESUMEN}

Objetivos: Determinar los niveles de inteligencia emocional predominantes en los estudiantes de la Facultad de Enfermería de la Universidad Nacional del Centro del Perú en el año 2013. Método: Se empleó el método descriptivo prospectivo. Se evaluaron un total de 195 estudiantes de 16 a 30 años de edad en toda la facultad. Para la recolección de datos, se aplicó el inventario Emocional de BarOn y el inventario de coeficiente emocional (I$\mathrm{CE})$ que permitió conocer su inteligencia emocional. Resultados: Se determinó los niveles de inteligencia emocional general siendo la capacidad emocional "adecuada" o promedio en un $49 \%$ seguido de una capacidad emocional "por debajo del promedio" y "muy bajo" con el $36 \%$ y 11 \% respectivamente. En cuanto a los componentes, intrapersonal, presenta una capacidad emocional adecuada del $83 \%$, siendo la más alta respecto a los demás componentes seguido del interpersonal $55 \%$ y el estado de ánimo $54 \%$, quienes presentaron inteligencia emocional adecuada o promedio, los estudiantes que alcanzaron una baja capacidad emocional por debajo del promedio fueron en los componentes de adaptabilidad $53 \%$, en manejo de estrés 42 $\%$. En relación al sexo masculino en el componente interpersonal y manejo de estrés presenta un $56 \%$ y $39 \%$ por debajo del promedio; en mujeres en el componente adaptabilidad y manejo de estrés con $54 \%$ y $44 \%$ presentan capacidad emocional debajo del promedio. Conclusión: Los componentes de inteligencia emocional general, adaptabilidad y manejo de estrés, tienen niveles de riesgo y deberán ser mejorados al igual que la inteligencia

emocional interpersonal en sexo masculino. No se encontraron diferencias significativas entre varones y mujeres en la inteligencia emocional general.

Palabras clave: Inteligencia emocional, estudiantes, enfermería.

1 Médico Cirujano, Magister en Gestión en Servicios de Salud, Ex Gerente Regional de Salud de Junín, Director del CAP II Chilca ESSALUD, investigador de la Universidad Nacional del Centro del Perú.

2 Dra. en Administración de la Educación, docente de pregrado y postgrado en las universidades Continental, y Peruana Los Andes, investigadora de la Universidad Nacional del Centro del Perú. 


\section{ABSTRACT}

Objectives: To determine the prevailing emotional intelligence levels in students from the Nursing Faculty, Universidad Nacional del Centro del Perú in 2013. Methods: Prospective descriptive method was used. A total of 195 16-30 year old students were assessed in the faculty. For data collection, the BarOn Emotional Inventory and the emotional coefficient inventory (ICE) which allow to know the emotional intelligence were applied. Results: The emotional intelligence general levels were determined, being the "right" emotional capacity or average at $49 \%$ followed by a "below average" and "very low" emotional capacity with $36 \%$ and $11 \%$ respectively. According to the components, intrapersonal, presents an adequate emotional capacity of $83 \%$, the highest one, followed by interpersonal at $55 \%$ and the mood at $54 \%$, who had adequate or average emotional intelligence, students with low emotional capacity below average were in the adaptability component at $53 \%$, stress management at $42 \%$. Regarding to men, the interpersonal and stress management components at $56 \%$ and $39 \%$ below average; women in the adaptability and stress management components at $54 \%$ and 44 $\%$ have emotional capacity below average. Conclusions: The emotional intelligence general components, adaptability and stress management, presents risk levels and they should be improved as well as the interpersonal emotional intelligence in men. No significant differences between men and women in the general emotional intelligence were found.

Keywords: Emotional intelligence, students, nursing.

\section{INTRODUCCIÓN}

En el escenario universitario de hoy, donde las realidades y estímulos resultan ser múltiples y complejos son diversos los factores que podrían influir en los resultados académicos del estudiante y por ende en su deserción/permanencia en las universidades.

Las tensiones de la vida moderna, la continua globalización de los conocimientos en el terreno individual, estudiantil, profesional, laboral, la presión del reloj, la exigencia de un constante perfeccionamiento, entre otros factores, son situaciones que tienden a alterar el estado emocional de la mayoría de los estudiantes, llevándolos al borde de sus propios límites físicos y psíquicos (1).

No podemos medir el éxito solamente por la capacidad intelectual, sino también por la capacidad emocional. Cualidades como la capacidad de entender los sentimientos propios, la habilidad de entender los sentimientos de los compañeros y el control de las emociones para lograr un fin, son más importantes a veces en un salón de clases que ser exitoso en todas las materias. Lo ideal para los seres humanos, es al canzar el equilibrio entre la capacidad intelectual y emocional. Mayer y Salovey definen a la inteligencia emocional como: "Un conjunto de habilidades que explican las diferencias individuales en el modo de percibir y comprender nuestras emociones. Más formalmente, la inteligencia emocional es la habilidad para percibir, valorar y expresar emociones con exactitud, la habilidad para acceder $y / o$ generar sentimientos que faciliten el pensamiento, para comprender emociones y razonar emocionalmente, y finalmente la habilidad para regular emociones propias y ajenas" (2).

En los informes de evaluaciones nacionales e internacionales donde los estudiantes peruanos obtuvieron tan bajas calificaciones, se asume como uno de los factores condicionantes de estos resultados a la incapacidad emocional de dichos estudiantes, que las causas más importantes son: la baja autoestima, las condiciones socio económicas de la familia y el clima de aula.

En Estados Unidos Salovey, Stroud, Woolery han demostrado que los alumnos universitarios con más IE informan menor número de síntomas físicos, menos ansiedad social y depresión y mejor autoestima, mayor 
satisfacción interpersonal, mayor utilización de estrategias de afrontamiento activo para solucionar sus problemas. Además cuando estos alumnos son expuestos a tareas estresantes de laboratorio, perciben los estresores como menos amenazantes y sus niveles de cortisol y presión sanguínea son más bajos (3).

Paralelamente en Australia Ciarrochi, Deane y Anderson; presentan evidencias de que los estudiantes universitarios con alta IE responden al estrés con menor depresión y desesperanza (4).

Por su parte en España, Fernández investiga acerca de los efectos de una adecuada inteligencia emocional sobre el rendimiento académico siendo estos relacionados de forma directamente proporcional. Encontrando cuatro áreas fundamentales en las que una falta de IE provoca o facilita la aparición de problemas entre los estudiantes estos problemas serian: déficit en los niveles de bienestar y ajuste psicológico del alumnado, disminución en la cantidad y calidad de las relaciones interpersonales, descenso del rendimiento académico, aparición de conductas destructivas y consumo de sustancias adictivas (5).

Villacorta E. en su tesis: "Inteligencia emocional y rendimiento académico en estudiantes de Medicina Humana de la Universidad Nacional de la Amazonía Peruana." Concluye: El nivel o cociente emocional (CE) o nivel de inteligencia emocional (I-CE) total o global (CET) que predomina en los estudiantes de la Facultad de Medicina Humana de la UNAP es el promedio o adecuado (CEP), seguido por el alto o muy alto (CEA) y finalmente por el bajo (CEB). Los resultados evidencian que en los estudiantes investigados predomina significativamente el nivel de inteligencia emocional promedio o adecuado. Así mismo los sujetos del presente estudio, exhibiendo puntajes que los ubican en la capacidad emocional adecuada o promedio (CEP) en cada uno de los componentes - dimensiones del cociente emocional (CE), evidencian mayores fortalezas en la dimensión estado de ánimo general (CEAG), seguida por la de adaptabilidad (CAD), continuando la intrapersonal (CIA) y manejo de estrés (CME), ubicándose al final la interpersonal (CIE). Los estudiantes de la Facultad de Medicina Humana de la UNAP, en términos generales, muestran potencialidades y fortalezas en las habilidades de los subcomponentes de optimismo (OP), solución de problemas $(\mathrm{SP})$, autoconcepto (AC), tolerancia al estrés (TE) y relaciones interpersonales (RI), evidenciando debilidad en habilidades específicas de felicidad (FE), flexibilidad (FL), control de impulsos (Cl), asertividad (AS) y empatía (EM). Se determinó también que existe una correlación significativa entre la inteligencia emocional y el rendimiento académico de los estudiantes materia de investigación (6).

Loly y López desarrollaron una investigación titulada: la autoestima y los valores organizacionales en estudiantes universitarios y estudiantes no universitarios de Lima, reportaron resultados que indican que los estudiantes universitarios le dan mayor importancia a los valores como servicio al cliente, la limpieza y la calidad del producto y menor importancia a la honradez, el respeto a la persona y a la responsabilidad; del mismo modo, los estudiantes no universitarios (egresados, graduados y postgraduados) le otorgan mayor importancia al servicio al cliente, limpieza y confianza y menor importancia a la honradez, puntualidad y responsabilidad. Esta misma jerarquía se observa cuando se establece la relación con la autoestima, sobre todo cuando la autoestima es muy baja, baja o intermedia; mientras que cuando la autoestima es alta o muy alta, se ubica en primer lugar la limpieza y mejora la puntualidad hasta ubicarse en el cuarto lugar en importancia (7).

Centeno realizó una investigación sobre la influencia del programa "Explorando mis Emociones" en la inteligencia emocional de estudiantes del sexto grado de primaria de un centro educativo en Huancayo, llegando a la conclusión que dicho programa influyó positivamente en los estudiantes, ayudando 
a desarrollar su inteligencia emocional (8).

Castillo realizó un análisis comparativo entre la inteligencia emocional de 500 alumnos con padres separados y no separados, para ello utilizó el inventario de inteligencia emocional de ICE Bar-On, concluyendo que existen diferencias altamente significativas en la Inteligencia Emocional entre alumnos del quinto año de secundaria con padres separados y no separados (9).

Javier $L$, lparraguirre $M$, en la investigación: "Inteligencia emocional en estudiantes de la Facultad de Ciencias de la Salud de la Universidad Peruana Los Andes" en Huancayo-Perú, llegan a concluir que los niveles de inteligencia emocional general es muy baja, con resultados semejantes en sus componentes intrapersonal, interpersonal, adaptabilidad manejo del estrés y estado de ánimo general, siendo las carreras profesionales con los más bajos niveles: Enfermería y Odontología, ubicándose preferentemente en el nivel adecuado las carreras de Farmacia y Bioquímica, Psicología y Obstetricia. No se encontraron diferencias significativas entre varones y mujeres en la inteligencia emocional general (10).

A través de este estudio se pretende resolver el siguiente problema: ¿Cuál es el nivel predominante de inteligencia emocional de los estudiantes de la Facultad de Enfermería de la Universidad Nacional del Centro del Perú en el año 2013?

El objetivo general fue, determinar el nivel predominante de inteligencia emocional de los estudiantes de la Facultad de Enfermería de la Universidad Nacional del Centro del Perú en el 2013; los objetivos específicos fueron: evaluar la inteligencia emocional de los estudiantes, analizar las características de la inteligencia emocional de los estudiantes, comparar la inteligencia emocional en los estudiantes en función a su género.

La importancia de este estudio radica en el hecho que ayudará en el proceso del crecimiento personal y social de toda la comunidad educativa, beneficiando a cada uno de los estudiantes. De igual forma, el programa de inteligencia emocional brinda herramientas al estudiante de enfermería para el control y manejo de la agresividad dándole importancia a los valores y al acatamiento de normas, porque al implantar este programa, la población atendida tendrá la oportunidad de aprender a manejar la agresividad evitando conductas violentas y permitiendo que se adopte la cultura de paz y convivencia universitaria como parte de su proceso social, lo cual, incidirá a nivel del entorno familiar de los estudiantes y a nivel de la población que circunda la institución educativa, aplicándolo en la actividad asistencial y comunitaria, de allí la relevancia práctica.

También la finalidad de esta investigación fue de contribuir al desarrollo de esta línea de trabajo con la intención de proporcionar nuevos conocimientos que sirvan de base empírica no solo a sus supuestos teóricos sino también a la praxis, que nunca debe dejarse en segundo lugar.

\section{MATERIAL Y MÉTODOS}

Se utilizó el método científico en la medida que se usó un proceso global y unitario con una secuencia de fases formalmente estructuradas en función de los objetivos de la investigación (11). Asimismo como método específico se utilizó el método descriptivo en la medida que se dedica a describir, analizar e interpretar sistemáticamente los datos obtenidos de la variable que se estudia, sin manipulación o tratamiento específico $(12,13)$. Anguera y otros, llama a este método: "No experimental" para enfatizar a la ausencia de la manipulación de variables independientes (11). El diseño utilizado fue no experimental, descriptivo comparativo, transversal.

Entre las técnicas e instrumentos de recolección de datos, se utilizó el cuestionario que permitió medir la inteligencia emocional a través del inventario de Bar-On (I-CE), creado por Reuven Bar-On para medir la inteligencia emocional, aplicable a sujetos de 
16 años a más, de administración individual y/o colectiva. Adaptada a nuestro medio por la Dra. Nelly Ugarriza Chávez (14).

\section{RESULTADOS}

\section{Resultados de Inteligencia Emocional}

Como puede observarse en la figura 1 se obtienen los resultados de Inteligencia Emocional General obtenidos mediante el inventario de Bar-On para los niveles de Inteligencia Emocional, Se tiene que el nivel adecuado alcanza un $49 \%$, seguido con una inteligencia emocional baja con 36 $\%$ y muy bajo con $11 \%$. El nivel inteligencia emocional buena presenta un mínimo porcentaje de $4 \%$.y el muy desarrollado 0 $\%$.

Se deduce que el mayor porcentaje de estudiantes tiene habilidad para adaptarse y enfrentar las demandas y presiones del medio.

En la figura 2 se aprecia que el IE del sexo masculino es adecuado en el $50 \%$ y baja también en $50 \%$ y en estudiantes del sexo femenino alcanza un nivel adecuado el 47 $\%$ una IE baja el $37 \%$ y una IE muy baja el $11 \%$, por otro lado la IE buena es del 5 $\%$. Indicándonos la necesidad de trabajar en ambos sexos con resultados bajos para mejorar las habilidades personales emocionales y sociales, para adaptarse y enfrentar las demandas y presiones de la profesión.

En la figura 3, respecto al componente intrapersonal se aprecia el nivel de inteligencia adecuada ocupa el mayor porcentaje con $83 \%$, le sigue el nivel muy bajo con $17 \%$. Es decir que la mayoría de los estudiantes están en contacto con sus sentimientos, se sienten bien acerca de si mismos y se sienten positivos en lo que están desempeñando.

En la figura 4, en relación al componente Intrapersonal según sexo, se observa que el $71 \%$ de estudiantes del sexo femenino de enfermería tienen una IE adecuada, le sigue una IE baja con el $18 \%$ una IE buena $8 \%$ y una IE muy baja con un $3 \%$. Así mismo se aprecia que el $83 \%$ de los alumnos varones tienen una IE adecuada y solo el $17 \%$ una IE baja. De lo que deducimos que la mayoría de las estudiantes son personas capaces de expresar abiertamente sus sentimientos, ser independientes, fuertes y confían en sus

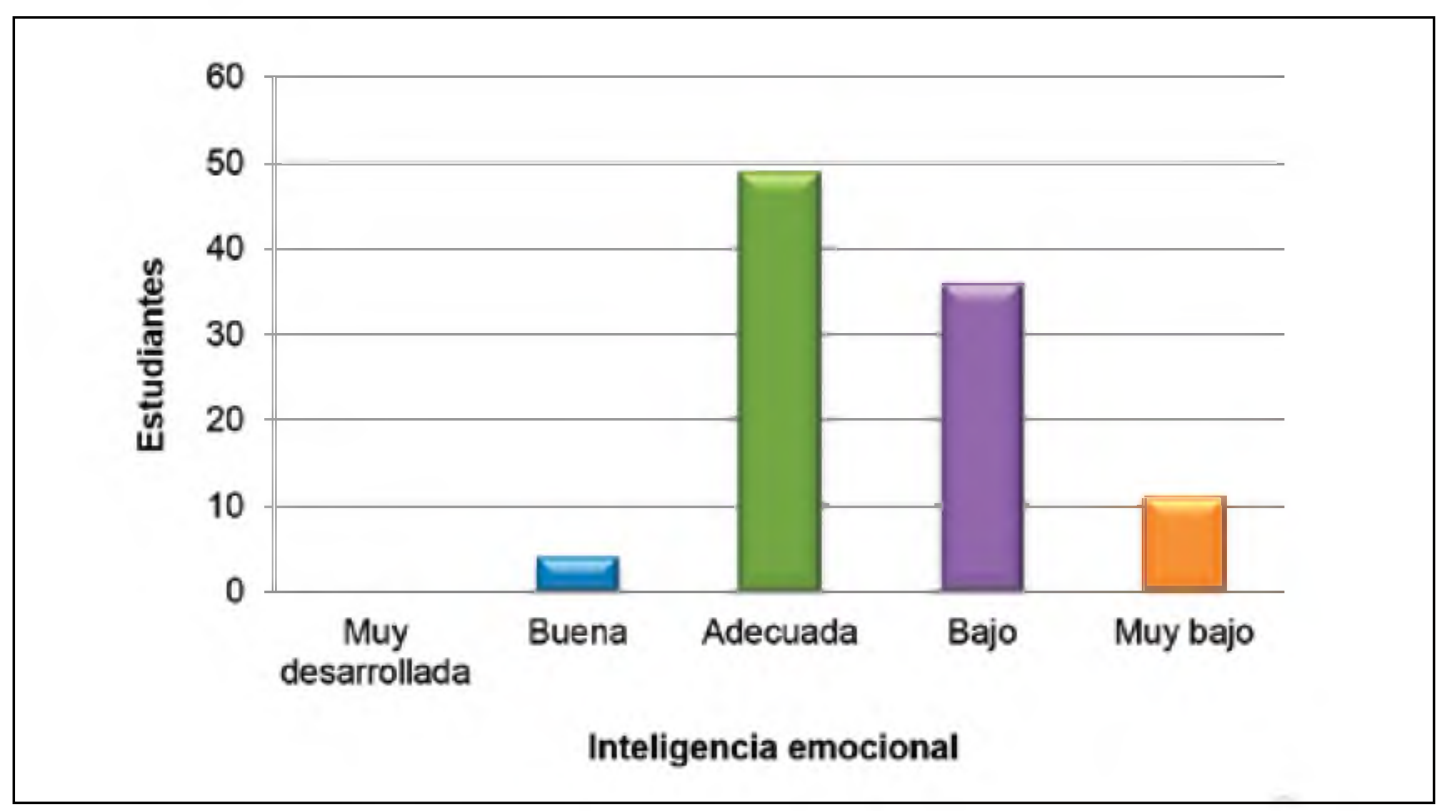

Figura $\mathrm{N}^{\circ}$ 1: Niveles de coeficiente de inteligencia emocional general de los estudiantes de Enfermería de la UNCP - 2013. 


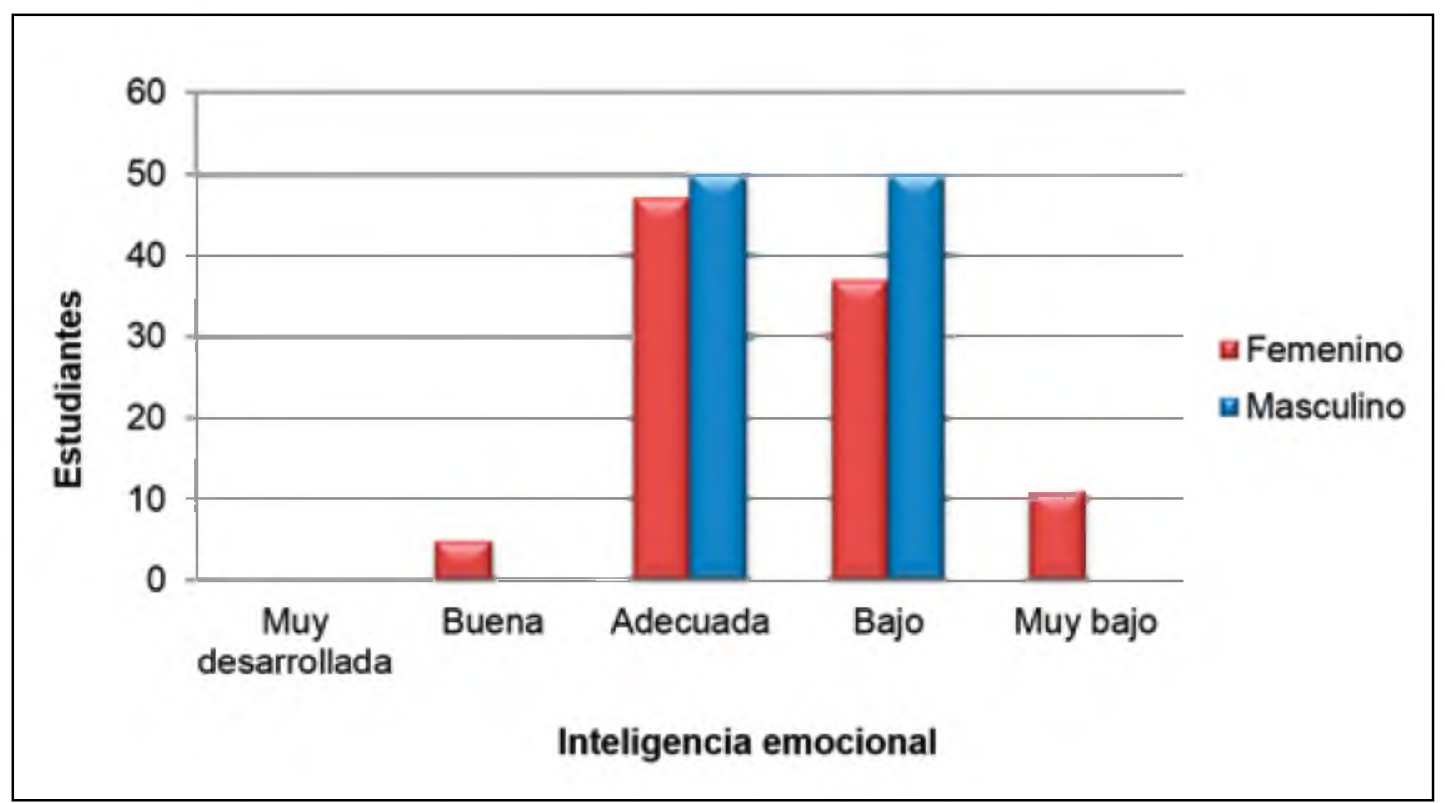

Figura $N^{\circ}$ 2: Niveles de coeficiente de inteligencia emocional según sexo de los estudiantes de Enfermería de la UNCP - 2013.

creencias.

En relación a la figura 5 se observan los niveles alcanzados en inteligencia interpersonal el nivel de IE adecuada presenta el porcentaje de $55 \%$ seguido de nivel, inteligencia emocional baja con 35 $\%$. El tercer lugar es el nivel muy bajo con 8 $\%$ le sigue el nivel de IE buena con $3 \%$. El porcentaje de IE adecuada nos indica que en general los estudiantes de enfermería son personas responsables y confiables que cuentan con habilidades sociales capaces de asumir responsabilidades orientadas a la comunidad.

En la figura 6, en relación a la Inteligencia Emocional interpersonal según sexo, observamos en los varones que el 56 $\%$ presenta una IE baja le sigue un 28 $\%$ con IE adecuada y un $16 \%$ de IE muy bajo. Lo que indica que los estudiantes del

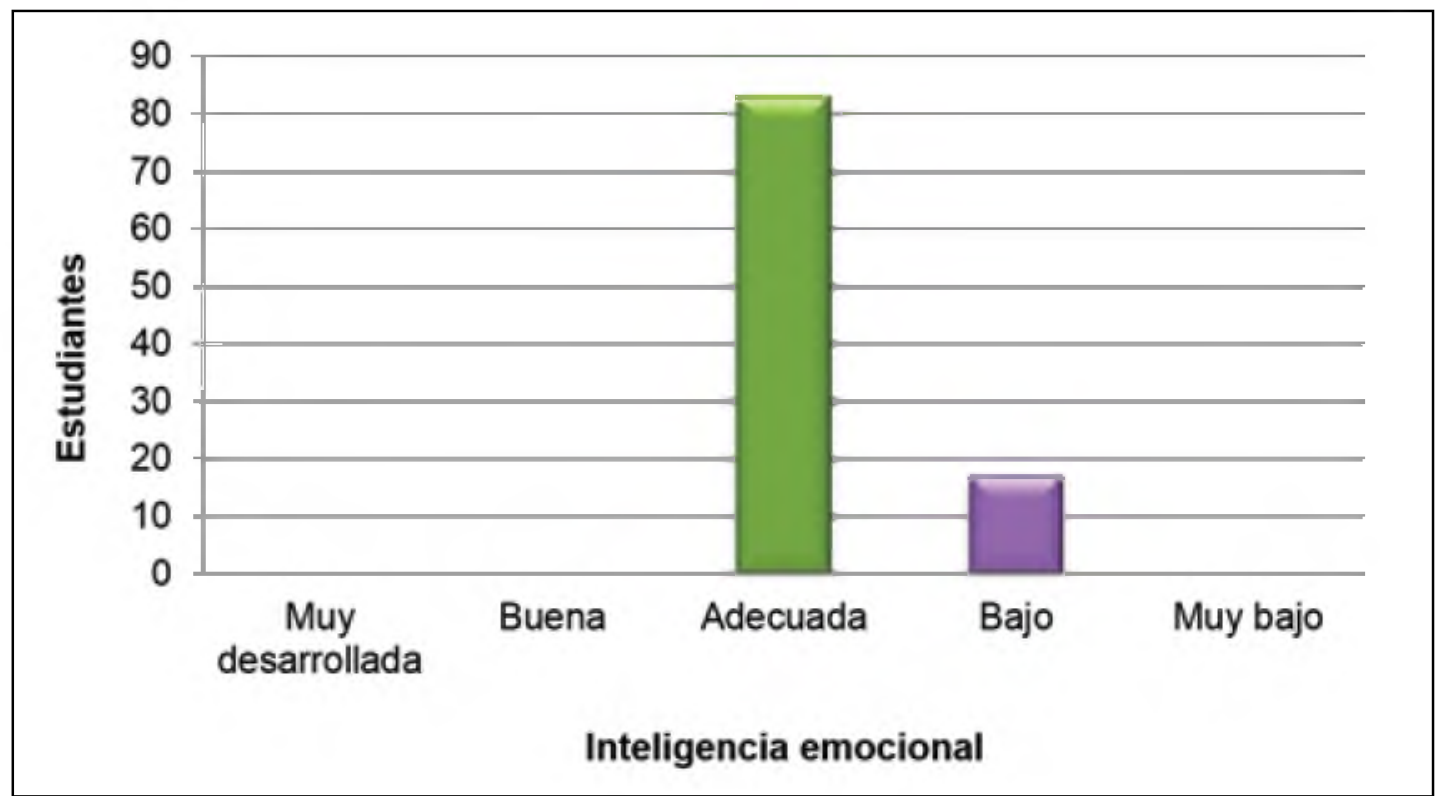

Figura $N^{\circ}$ 3: Niveles de inteligencia intrapersonal en los estudiantes de Enfermería de la UNCP - 2013. 


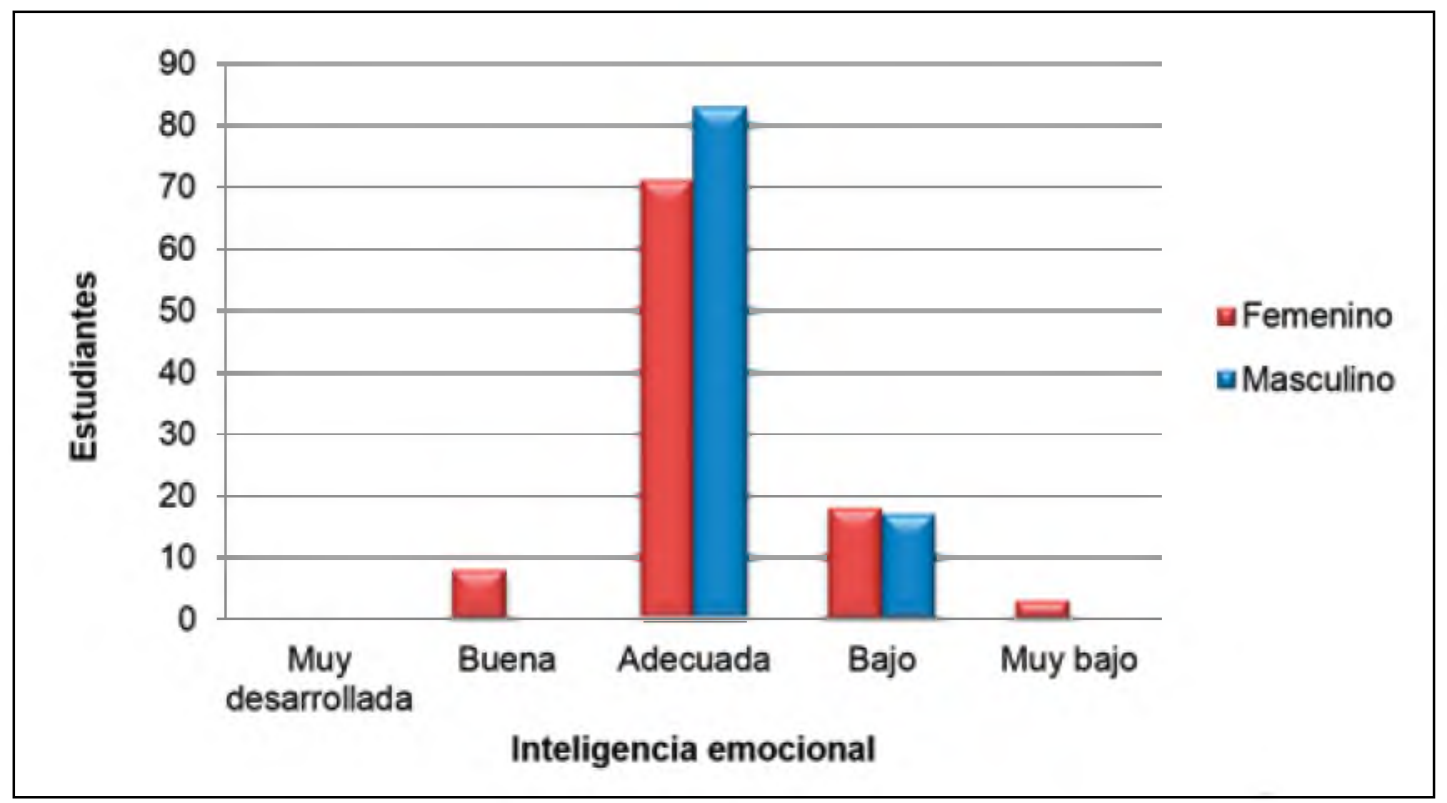

Figura $\mathrm{N}^{\circ}$ 4: Niveles de inteligencia intrapersonal según sexo en los estudiantes de Enfermería de la $\mathrm{UNCP}-2013$.

sexo masculino de enfermería tienen que mejorar algunos subcomponentes como la empatía, las relaciones interpersonales y responsabilidad social. En las mujeres se puede apreciar que el $56 \%$ de las estudiantes de enfermería tiene una IE interpersonal adecuada, un $33 \%$ IE interpersonal baja y una IE interpersonal de $7 \%$ muy baja, asimismo un IE interpersonal de $4 \%$ buena. Podemos deducir que la mayoría de las estudiantes muestran características de responsabilidad y confiabilidad.

En relación a la figura 7 , los niveles de inteligencia adaptabilidad. El nivel de inteligencia bajo ocupa el mayor porcentaje con $53 \%$, le sigue el nivel, inteligencia emocional adecuado con $38 \%$. El tercer lugar es el nivel muy bajo con $8 \%$. El nivel muy desarrollado. Estos resultados nos indican que los estudiantes necesitan

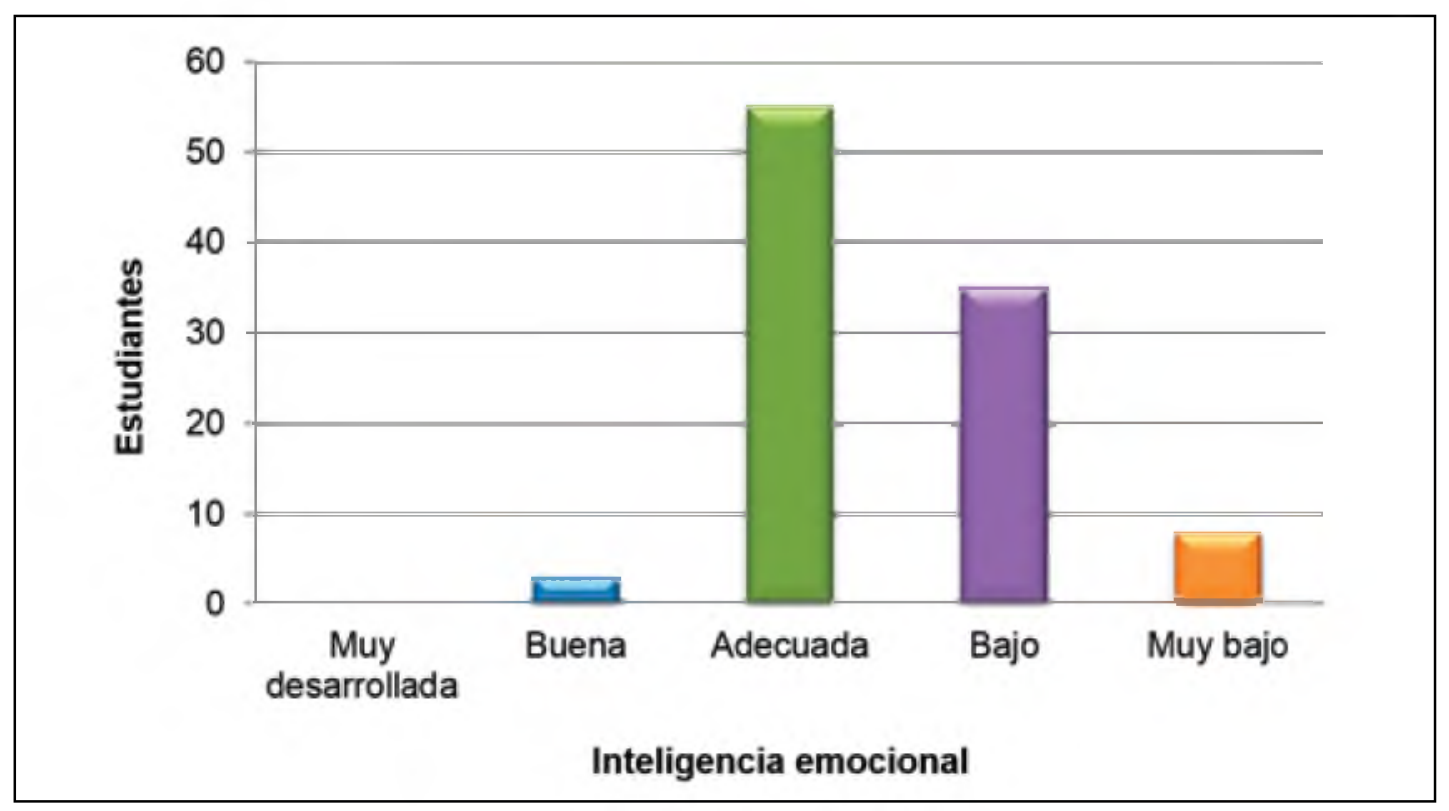

Figura $N^{\circ}$ 5: Niveles de inteligencia interpersonal en estudiantes de Enfermería de la UNCP - 2013. 


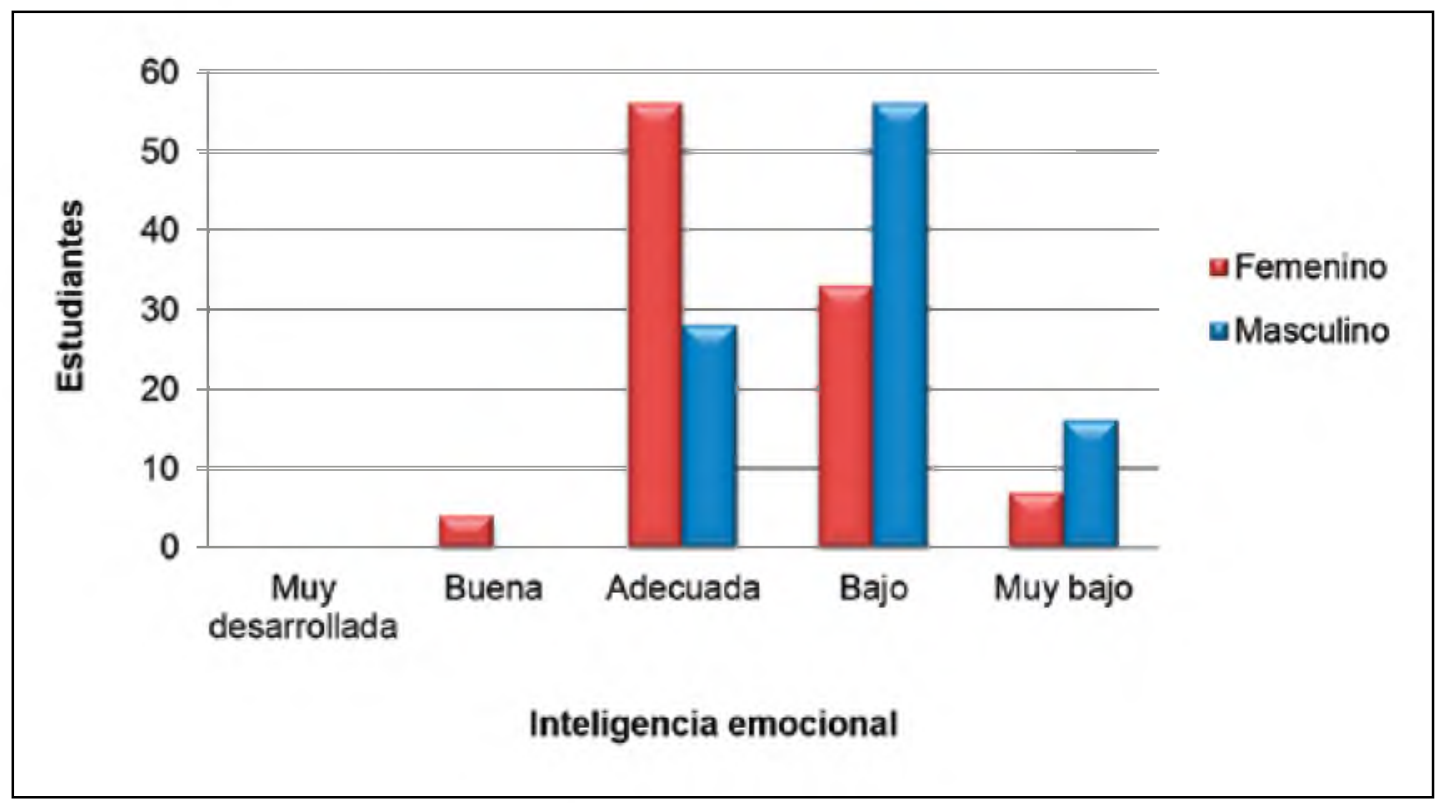

Figura $N^{\circ}$ 6: Niveles de Inteligencia Interpersonal según sexo en estudiantes de Enfermería de la UNCP. 2013.

adecuarse a las exigencias del entorno y efectivizar la solución de problemas.

En relación a la figura 8 , los niveles de inteligencia adaptabilidad según sexo, se puede apreciar que los estudiantes del sexo masculino alcanzan un $50 \%$ de IE de adaptabilidad adecuada y un $50 \%$ baja. Lo que nos indica que la mitad de los estudiantes varones se pueden adecuar a las exigencias del entorno y pueden resolver problemas de manera efectiva. Así mismo se puede observar que el $54 \%$ de las alumnas de enfermería tiene una IE de adaptabilidad baja, le sigue otro grupo con $36 \%$ adecuada, un $9 \%$ muy baja y un $1 \%$ de IE de adaptabilidad buena. De lo que podemos deducir que la mayoría de las estudiantes no se adecuan a las exigencias del entorno y no saben resolver problemas en forma efectiva.

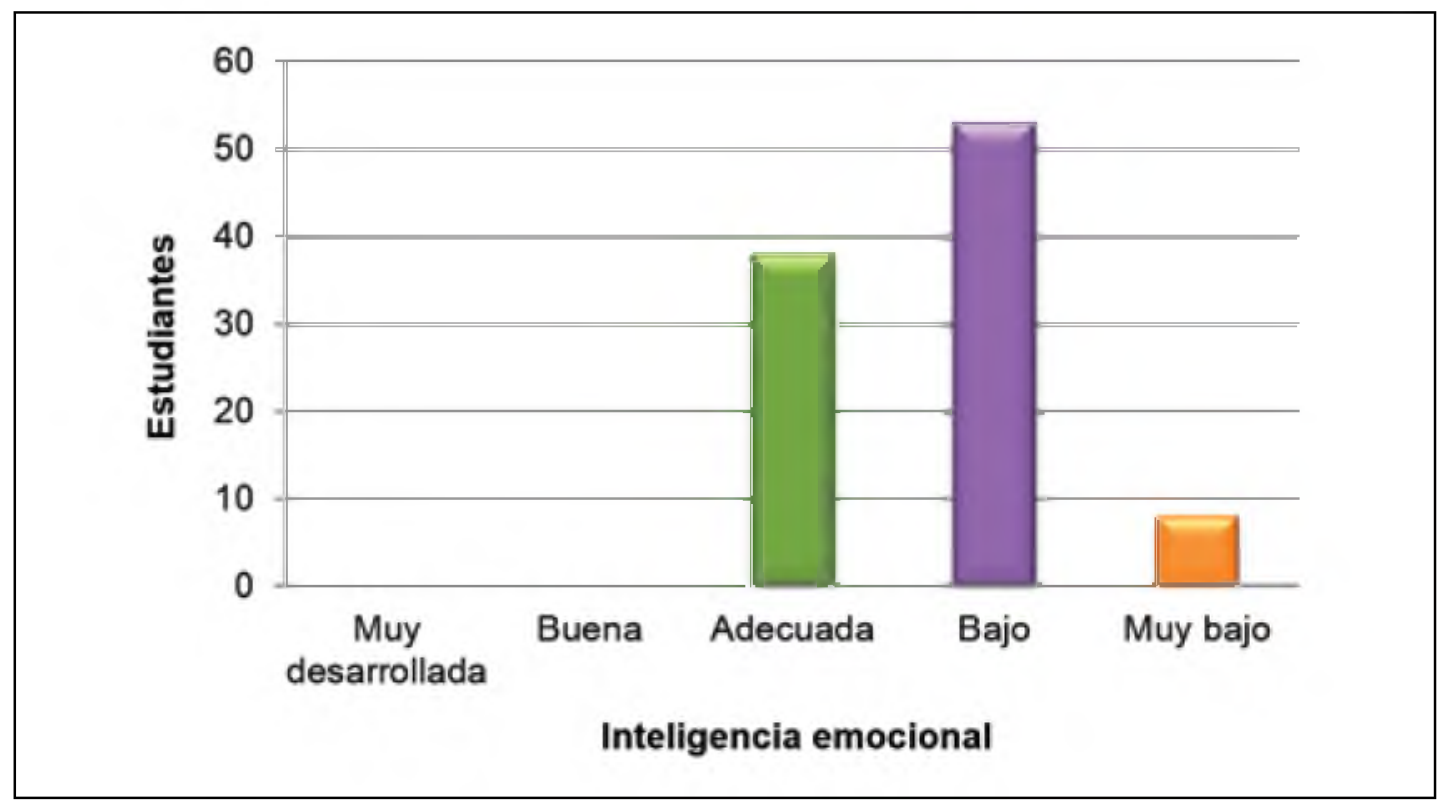

Figura $N^{\circ}$ 7: Niveles de inteligencia adaptabilidad en estudiantes de Enfermería de la UNCP - 2013. 


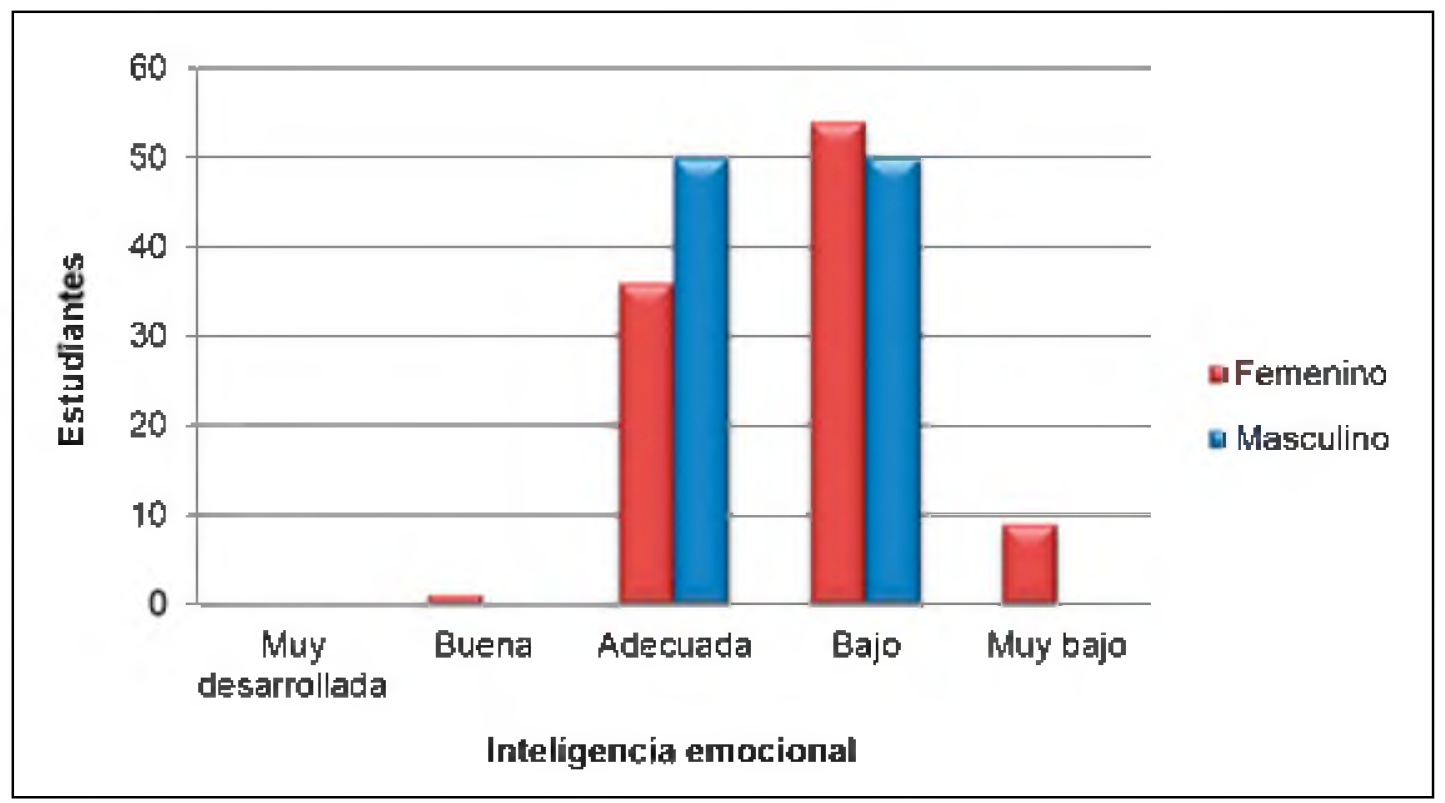

Figura $N^{\circ}$ 8: Niveles de Inteligencia Adaptabilidad según sexo en estudiantes de Enfermería de la UNCP $-2013$.

En relación a la figura 9 , se observan los niveles alcanzados en el componente manejo del estrés. El nivel de inteligencia emocional bajo ocupa el mayor porcentaje con $42 \%$, le sigue el nivel, inteligencia emocional adecuada con $41 \%$. El tercer lugar, es nivel muy bajo con $16 \%$, con solo $2 \%$ se tiene un nivel de inteligencia buena y $0 \%$ I.E, muy desarrollada. De lo que deducimos al sumar el porcentaje bajo y muy bajo de 58 \% que la mayoría de los estudiantes no es capaz de resistir a la tensión sin perder el control y no es capaz de trabajar bajo presión.

En la figura 10, los niveles de inteligencia manejo de estrés, según sexo, se aprecia que los estudiantes del sexo masculino tienen un $39 \%$ de IE adecuada, un $39 \%$ baja y un $22 \%$ de IE muy baja. Al sumar los niveles bajo y muy bajo alcanzan un $61 \%$, de lo que deducimos que no tienen

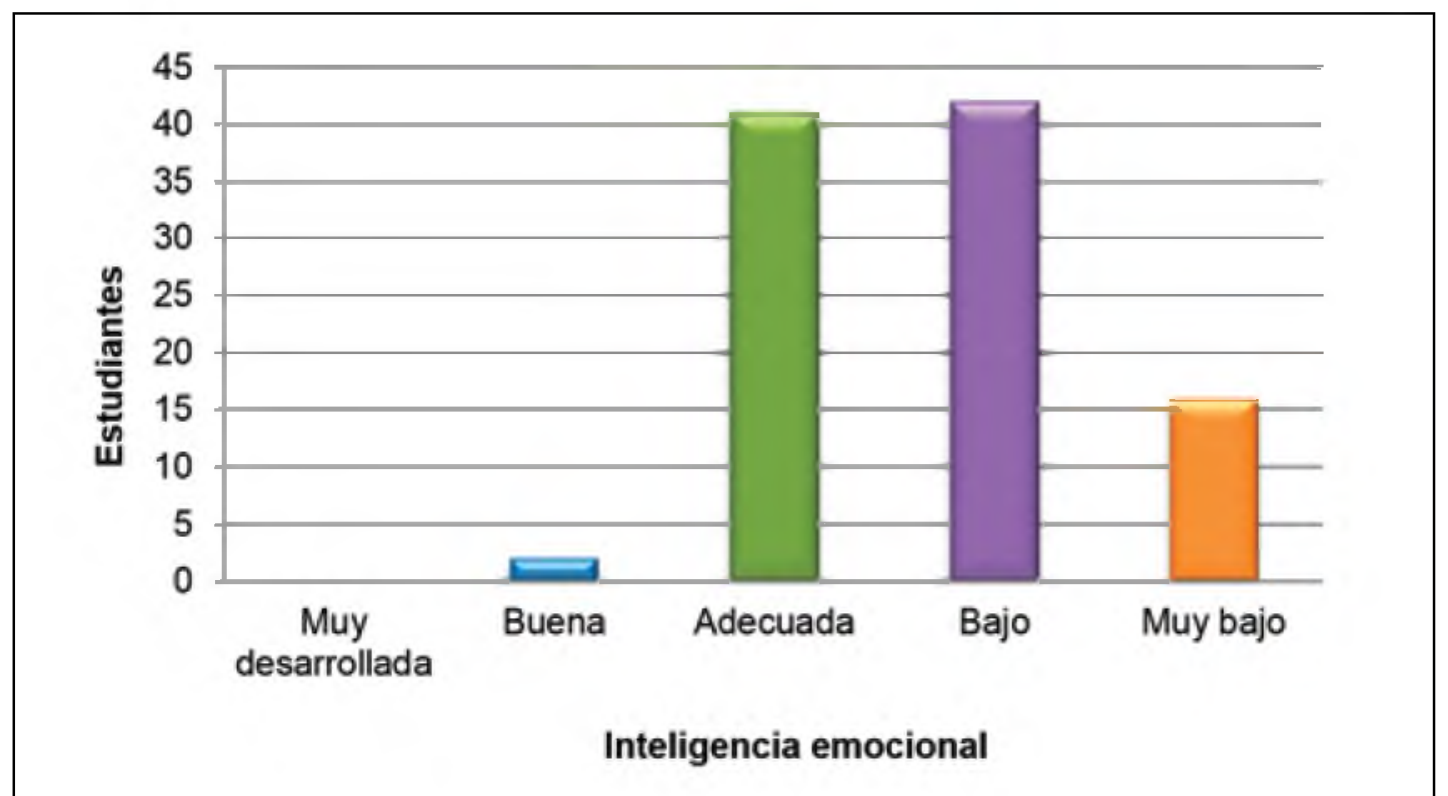

Figura $N^{\circ}$ 9: Niveles de inteligencia manejo del estrés en estudiantes de Enfermería de la UNCP - 2013. 


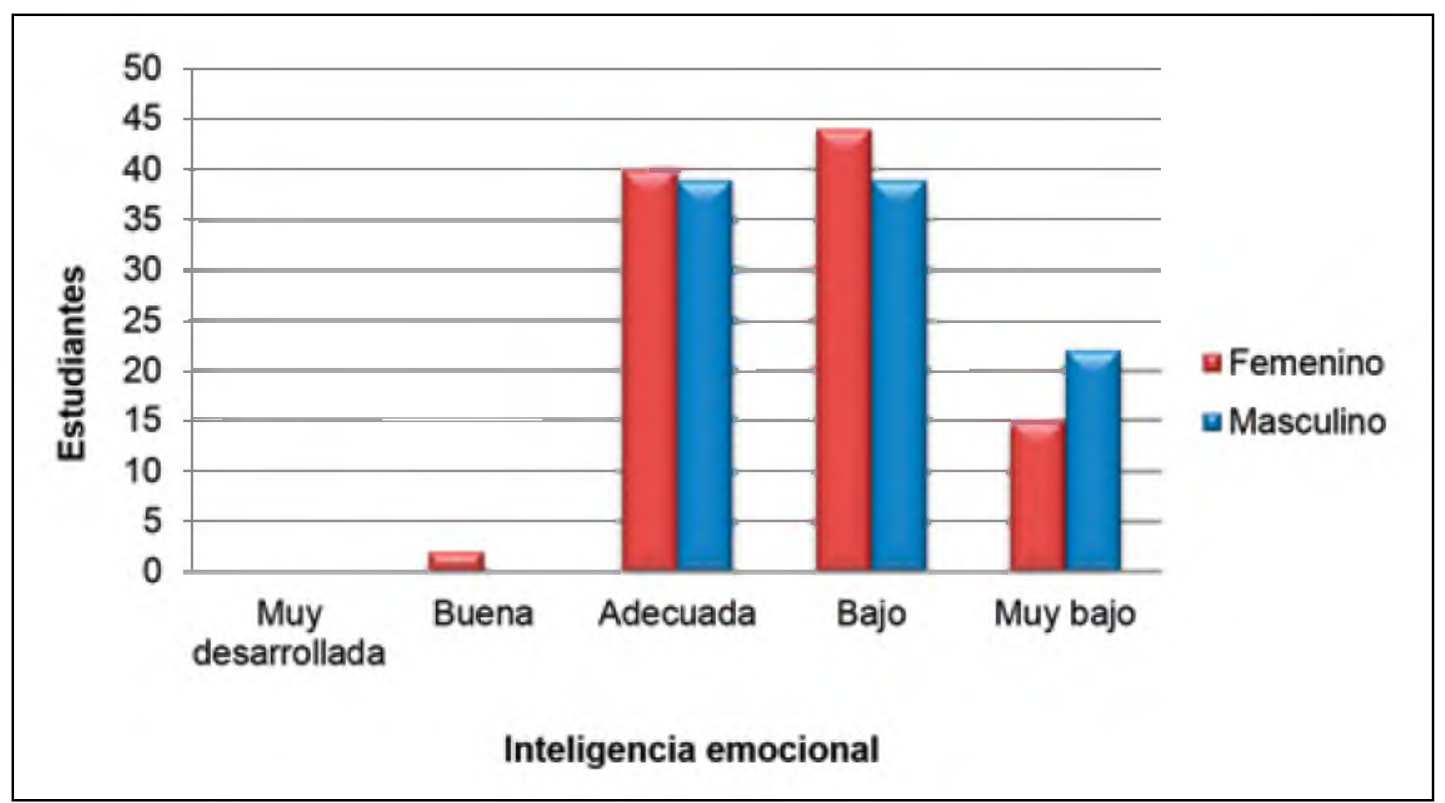

Figura $N^{\circ}$ 10: Niveles de inteligencia emocional manejo de estrés según sexo en estudiantes de Enfermería de la UNCP - 2013.

tolerancia al estrés y no controlan sus impulsos. En relación a los estudiantes del sexo femenino alcanzan una IE de $44 \%$ baja; un $40 \%$ adecuada, le siguen un $15 \%$ muy baja y un $2 \%$ buena. Al sumar los niveles bajo y muy baja estos alcanzan un $59 \%$ de lo que deducimos que la mayoría de las estudiantes no son tolerantes al estrés y no pueden controlar sus impulsos.
En relación a la figura 11, los niveles de Inteligencia Estado de Animo General según sexo se puede observar que el $56 \%$ de estudiantes varones tienen IE adecuado, y un $44 \%$ y de IE bajo. Cifras que nos dicen que la mayoría de los estudiantes de sexo masculino tiene un sentido de felicidad y optimismo. Así mismo evidenciamos en los estudiantes de sexo femenino un $55 \%$

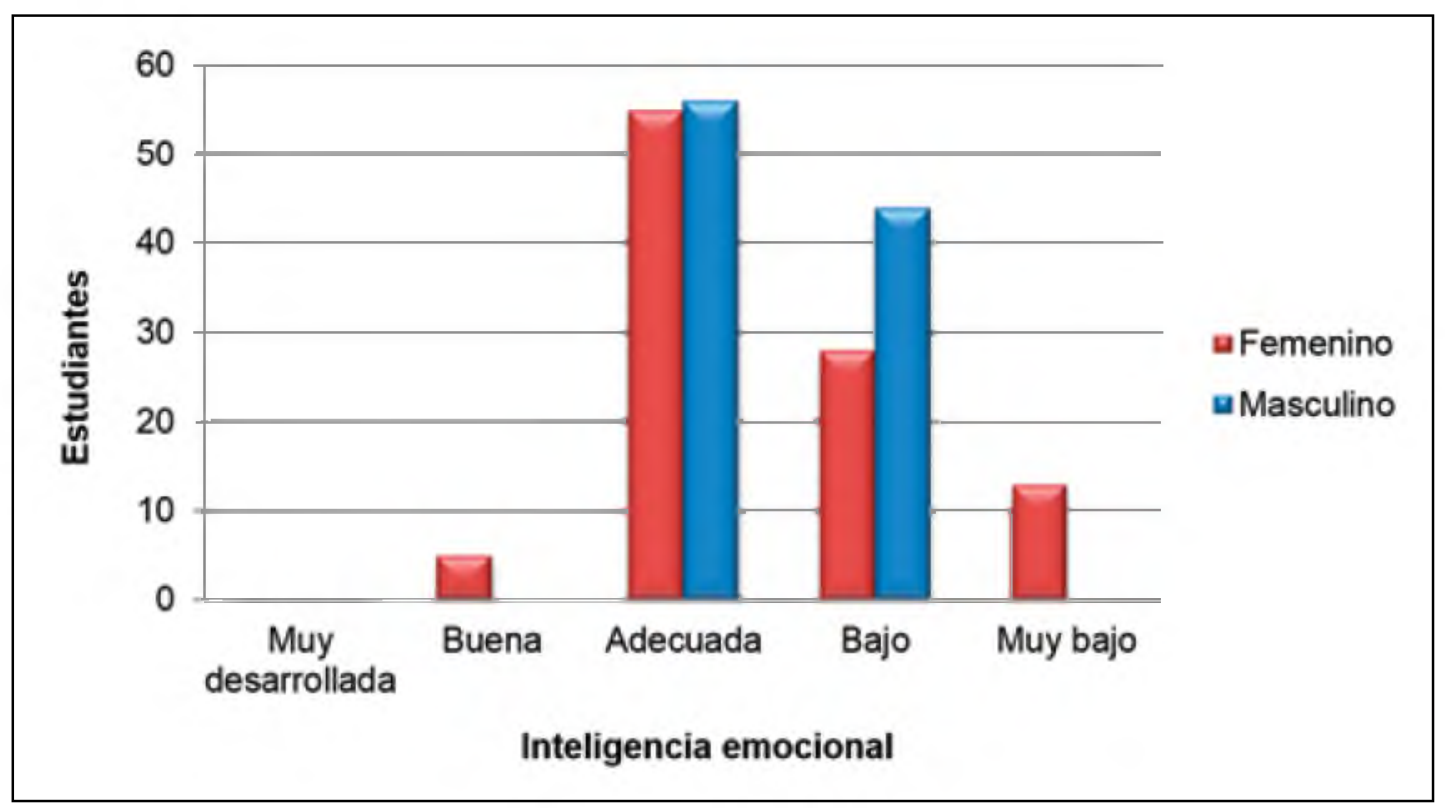

Figura $\mathrm{N}^{\circ}$ 11: Niveles de Inteligencia estado de ánimo general según sexo en estudiantes de Enfermería de la UNCP - 2013. 
de IE de estado de ánimo adecuado; un $28 \%$ bajo, un $13 \%$ muy bajo y un $5 \%$ IE de estado de ánimo buena. De lo que deducimos que las estudiantes poseen las capacidades de disfrutar la vida y visión de optimismo.

\section{DISCUSIÓN}

Los resultados antes expuestos presentan el siguiente panorama. En la figura 1 se tiene que el nivel adecuado alcanza un $49 \%$, seguido con una inteligencia emocional baja con $36 \%$ y muy bajo con $11 \%$. El nivel inteligencia emocional buena presenta un mínimo porcentaje de $4 \%$. Esto sugiere que la capacidad para percibir, asimilar, comprender y regular las propias emociones y las de los demás está en promedio adecuado, sin embargo existe un porcentaje preocupante al sumar el nivel bajo y muy bajo $47 \%$. Esto indica que la capacidad para percibir, asimilar, comprender y regular las propias emociones y las de los demás está en riesgo (15) por lo que posiblemente los beneficios que proporciona una adecuada inteligencia emocional (3) como: menor número de síntomas físicos, menos ansiedad social y depresión y mejor autoestima, mayor satisfacción interpersonal, mayor utilización de estrategias de afrontamiento activo para solucionar sus problemas Salovey, Stroud, Woolery también tendrían pocas probabilidades de estar presentes en los estudiantes de la Facultad en estudio. Sin embargo, son éstas las cualidades que requieren los futuros profesionales que atenderán la salud de personas de alta sensibilidad emocional, debido a la vulnerabilidad de su salud.

Siguiendo con el análisis de la inteligencia emocional, se encuentra que en la inteligencia intrapersonal existe un $83 \%$ en el nivel adecuado (figura 3). Ello indica que la comprensión de sí mismo, el auto concepto y la independencia, forman parte de su inteligencia emocional en el desarrollo de los actuales estudiantes de Facultad de Enfermería. Por tanto, en la Facultad, sucedería lo opuesto a lo que dice Fernández (2004), que se facilitaría la aparición de problemas entre los estudiantes, y que éstos problemas serian: déficit en los niveles de bienestar y ajuste psicológico del alumnado, disminución en la cantidad y calidad de las relaciones interpersonales, descenso del rendimiento académico, aparición de conductas destructivas y consumo de sustancias adictivas (5). Los estudiantes de la Facultad de Enfermería maneja una adecuada IE intrapersonal que permite mirar el futuro con proyección de acuerdo a lo planteado por un representante del Banco mundial quien hizo mención que las habilidades cognitivas más las socio afectivas y equilibrio emocional son las cualidades de los profesionales con mayor empleabilidad y mejor pagadas (16).

En la inteligencia interpersonal se aprecia en toda la muestra que el nivel adecuada obtiene el $55 \%$ (figura). Por lo que si podrían desarrollar su capacidad empática; es decir, la habilidad para poder percatarse, comprender y apreciar los sentimientos de los demás, así como la facilidad para establecer y mantener relaciones interpersonales satisfactorias, ser cooperativo identificarse en grupo como un miembro constructivo (17). Todas ellas habilidades emocionales muy importantes en profesionales de enfermería que no solo aseguran una relación saludable con los demás sino que también benefician enormemente la salud física y emocional de ellos mismos. Por ello, la importancia de la psicoinmunología que estudia los vínculos entre el sistema inmunológico y el sistema nervioso central (18).

En lo correspondiente al componente adaptabilidad, el panorama es de $38 \%$ en el nivel adecuado y el nivel bajo $53 \%$ es preocupante. De acuerdo a Ugarriza las personas con puntuaciones altas en esta sub escala poseen tres características importantes: son realistas, flexibles y poseen capacidad para detectar y solucionar problemas en vez de evitarlos. Características muy importantes en todo profesional, pues la flexibilidad implica adecuar las emociones, sentimientos y 
comportamientos a situaciones cambiantes (17) ello en el caso de enfermería no garantizaría, la facilidad para responder adecuadamente a las exigencias del entorno.

En el componente manejo del estrés, se puede observar que el nivel bajo obtiene el porcentaje más alto con $42 \%$, le sigue el nivel adecuado con $41 \%$ y muy bajo con 16 $\%$, aun cuando el nivel adecuado suba un lugar este componente, no se puede dejar de reconocer que los porcentajes obtenidos en los niveles bajos siempre superan a los niveles superiores de inteligencia emocional en la muestra. La ubicación mayoritaria de la muestra se encuentra en los dos primeros niveles de inteligencia emocional: muy baja y baja. Ello indicaría que dichos estudiantes tendrían dificultades en el manejo del estrés, poca tolerancia a la frustración y altos niveles de ansiedad que pueden desencadenar el curso de una enfermedad (18). Y a decir de Plutchik (1980) si la ansiedad creada por el conflicto es prologada puede acabar creando no solo una gran tensión, sino también puede quebrar o trastornar la salud mental (19). Tales son los riegos a los que están expuestos de nuestros estudiantes, si no se brindan técnicas para el adecuado manejo del estrés. Sin embargo el Adecuado manejo del estrés que posee el $41 \%$ de los estudiantes implica adecuada capacidad para resistir los eventos adversos en situaciones estresantes, que una de las cualidades más importantes en los profesionales actuales y que requiere el mercado laboral (20).

El componente estado de ánimo general presenta en los estudiantes de la Facultad de Enfermería el siguiente panorama: la inteligencia emocional adecuada ocupa el $54 \%$, le sigue la baja con un $30 \%$ y muy baja con $11 \%$. Las principales características del estado de ánimo general son la felicidad y el optimismo lo que distinguirá a la mayoría de los estudiantes de la Facultad de Enfermería. La primera característica permite ver a las personas con una disposición feliz y es agradable estar con ellos. La segunda implica una actitud positiva aun en las adversidades.
Por otro lado, se comparó la inteligencia emocional de varones y mujeres de la muestra encontrándose valores de Inteligencia emocional parecidos en todos los componentes a diferencia en los componentes interpersonal y adaptabilidad. En el componente interpersonal los varones obtienen un $28 \%$ de IE adecuada y 56 $\%$ baja y $16 \%$ muy baja en relación con las estudiantes del sexo femenino que obtienen un $56 \%$ de IE adecuada $33 \%$ baja y un $7 \%$ muy baja, es decir que las mujeres presentarían un grado mayor de confiabilidad y de responsabilidad en comparación con los estudiantes varones (figura 6). Por otro lado en el componente de adaptabilidad ocurre lo contrario los varones obtienen el $50 \%$ de IE adecuada y $50 \%$ baja y las estudiantes del sexo femenino alcanzan una IE de $36 \%$ adecuada y un 54 $\%$ de IE baja (figura 8). Lo que significaría que los estudiantes del sexo masculino se adecuan mejor a las exigencias del entorno y pueden lidiar de manera efectiva a las situaciones problemáticas según sostiene Baron.

Las conclusiones a las cuales se arribaron son las siguientes:

1. El nivel de inteligencia emocional general predominante en los estudiantes de la Facultad de Enfermería de la Universidad Nacional del Centro del Perú es el adecuado en un $49 \%$ y con $47 \%$ bajo y muy bajo lo cual sugiere que la capacidad para percibir, asimilar, comprender y regular las propias emociones y las de los demás, tan importante en profesionales de la salud, está en riesgo en un $47 \%$ de los encuestados.

2. La inteligencia intrapersonal adecuada alcanza un 83 \% en toda la muestra lo que indica que son capaces de expresar abiertamente sus sentimientos y ser independientes.

3. En relación al componente interpersonal el $55 \%$ alcanza un IE adecuada, pero hay un nivel preocupante de IE baja y muy baja que sumado alcanza el $43 \%$. 
Lo que indica que en su mayoría tienen responsabilidad social y relaciones interpersonales. Sin embargo un grupo significativo tendrá que trabajar en mejorar este componente.

4. En relación al componente de adaptabilidad solo se alcanza el 38 $\%$ de IE de adaptabilidad adecuada y el $60 \%$ con nivel de inteligencia bajo y muy bajo lo que indica que no se adecuan a las exigencias del entorno y no solucionan adecuadamente los problemas.

5. En relación al componente de manejo del estrés la IE para manejo de estrés adecuada es de $41 \%$ pero al sumar el nivel de IE baja y muy baja es de $58 \%$ lo que nos indica que no son tolerantes al estrés y tampoco tienen control de sus impulsos.

6. En relación al componente de estado de ánimo el nivel de IE de estado de ánimo adecuado alcanza el $54 \%$ lo que nos indica la capacidad de las estudiantes para disfrutar la vida y mirar con optimismo el futuro.

7. Los estudiantes varones y mujeres no difieren significativamente en sus niveles de inteligencia emocional a excepción en el componente de interpersonal en la que los varones alcanzan un IE bajo y muy bajo de $72 \%$ en relación al 56 $\%$ de las estudiantes del sexo femenino; lo que indicaría que las estudiantes serían personas más responsables y confiables.

\section{REFERENCIAS BIBLIOGRÁFICAS}

1. López M. La inteligencia emocional y las estrategias de aprendizaje como predictores del rendimiento académico en estudiantes universitarios. [Tesis de maestría]. Lima: Universidad Nacional Mayor de San Marcos; 2008.

2. GROP (Grup de Recerca en Orientació
Psicopedagògica) [Internet]. España: Rafael Bisquerra Alzina; [citado el 10 de abril de 2013]. La inteligencia emocional según Salovey y Mayer; [1 pantalla]. Disponible en: http://www. rafaelbisquerra.com/es/inteligenciaemocional/inteligencia-emocionalsegun-salovey-mayer.html

3. Salovey P, Stroud L, Woolery A, Epel E. Perceived emotional intelligence, stress reactivity, and symptom reports: Further explorations using the trait meta-mood scale. Psychology and Health. 2002; 17(5): $611-627$.

4. Ciarrochi J, Deane F, Anderson S. Emotional intelligence moderates the relationship between stress and mental health. Personality and Individual Differences. 2002; 32(2): 197-209.

5. Extremera N, Fernández $\mathrm{P}$ El papel de la inteligencia emocional en el alumnado: evidencias empíricas. Revista Electrónica de Investigación Educativa, 2004; 6(2): $1-14$.

6. Villacorta E. Inteligencia emocional y rendimiento académico en estudiantes de Medicina Humana de la Universidad Nacional de la Amazonía Peruana. [Tesis doctoral]. Lima: Universidad Nacional Mayor de San Marcos; 2010.

7. Loly A, López E, Atalaya M. La autoestima y los valores organizacionales en estudiantes universitarios y no universitarios de Lima. Rev. investig. psicol. 2002; 5(1): 141-155

8. Centeno C. Influencia del Programa Explorando mis emociones en la inteligencia emocional de estudiantes del sexto grado de primaria de un Centro Educativo en Huancayo. [Tesis de pregrado]. Huancayo: Universidad Peruana Los Andes; 2007.

9. Castillo R. Análisis comparativo de los niveles de la inteligencia emocional en los alumnos del quinto año de secundaria con padres separados y no separados del Colegio Nacional Mixto Cartavio. [Tesis de pregrado]. Trujillo: Universidad Cesar Vallejo; 2005.

10. Javier L, Iparraguirre M. Inteligencia emocional en estudiantes de la facultad de Ciencias de la salud de la Universidad Peruana Los Andes. Rev. 
innov. 2012; (13): 121-128.

11. Anguera T. Métodos de investigación en Psicología. $1^{\underline{a}}$ ed. Madrid: Síntesis; 1995.

12. Sánchez H. Reyes C. Metodología y diseños de la investigación científica. $2^{\underline{a}}$ ed. Lima: Mantaro; 1996.

13. Fox J David. El proceso de investigación en educación. $2^{\underline{a}}$ ed. Pamplona: Universidad de Navarra Ediciones S.A; 1981.

14. Ugarriza N. La evaluación de la inteligencia emocional a través del inventario de BarOn (I-CE) en una muestra de Lima Metropolitana. Persona. 2001; (4): 129-160. Disponible en: http://www.redalyc.org/ articulo.oa? id = 147118178005

15. Salovey P, Mayer J. Las emociones factor determinante. Barcelona: Peña; 2004.

16. Pérez N, Castejón J. Diferencias en inteligencia emocional en estudiantes universitarios. Análisis en función de la titulación y el género. España: Universidade da Coruña; 2009: Disponible en: http://www.educacion. udc.es/grupos/gipdae/documentos/ congreso/NIIlcongreso/pdfs/165.pdf

17. Ugarriza N. La Evaluación de la Inteligencia Emocional a través del inventario de BarOn (I-CE) en una Muestra de Lima Metropolitana. $1^{1 \mathrm{a}} \mathrm{ed}$. Lima: Libro Amigo; 2001.

18. Goleman D. Inteligencia Emocional. 19 ed. Argentina: Javier Vergara; 1998.

19. Plutchik P Emotion. $1^{\mathrm{a}}$ ed. New York: Harper and Row; 1980.

20. Kudo I. Fortaleciendo las habilidades y la empleabilidad en el Perú. Encuentro de la Calidad en la Universidad Peruana. Perú: Universidad Nacional de Trujillo; 2011. 